\title{
Prell, Uwe (2020): Die Stadt. Eine Einführung für die Sozialwissenschaften
}

\author{
Heike Herrmann
}

Eingegangen: 3. März 2021 - Angenommen: 5. März 2021 - Online veröffentlicht: 21. April 2021

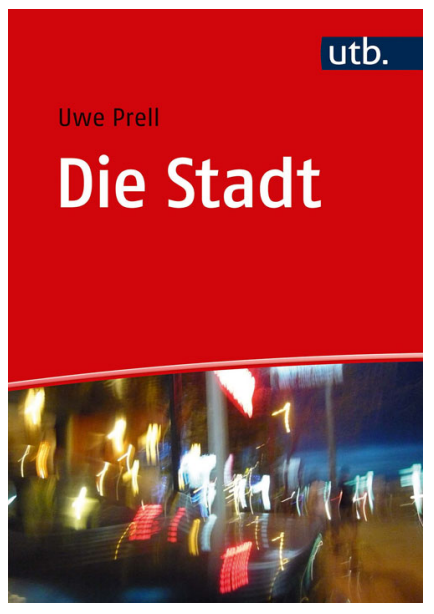

Obwohl der Titel zunächst recht schlicht daherkommt, so ahnen Leserin und Leser gleich, dass die vorliegenden knapp 150 Seiten im Taschenbuch-Format eigentlich nicht ausreichen können, um die Stadt zu behandeln. Uwe Prell fokussiert seine Überlegungen entsprechend auf - und dies ist ungewöhnlich - die Suche nach der Antwort auf eine oder (genau genommen) zwei Fragen, die sich durch das gesamte Buch ziehen: „Für welches Problem ist die Stadt eine Lösung?“ (S. 5) oder ,Auf welche Frage ist die Stadt eine Antwort?" (S. 79). Der Hintergrund dieser Leitfragen erschließt sich nicht sofort. Im Verlauf des Buches wird jedoch deut-

Prof. Dr. Heike Herrmann, Fachbereich Sozialwesen, Hochschule Fulda, Leipziger Straße 123, 36037 Fulda heike.herrmann@sw.hs-fulda.de

$$
\text { (c) (1) }
$$

(c) 2021 Herrmann; licensee oekom verlag. This Open Access article is published under a Creative Commons Attribution 4.0 International License. lich, dass die von Uwe Prell in seine Überlegungen einbezogenen Disziplinen verschiedene Antworten anbieten bzw. Fragen stellen und dass ihr Gemeinsames eben die Tatsache ist, dass Wissenschaft vor allem Fragen stellt und eindeutige Antworten eher selten zu geben sind. Entsprechend wenig eindeutig, das ist eine wesentliche vermittelte Erkenntnis des Buches, ist auch die Stadt zu betrachten.

$\mathrm{Zu}$ Beginn führt Prell zunächst allgemein in seine interdisziplinäre Betrachtung ein und bringt uns beim Lesen gleichzeitig seine gewählte Ausdrucksweise näher. Die Stadt wird als Form und Werkzeug zur Steigerung der menschlichen Möglichkeiten vorgestellt. In dem ersten umfangreicheren Kapitel zur Theorie wählt der Autor drei Zugänge, um sich der Stadt zu nähern: Er pointiert die Sicht auf die Stadt aus unterschiedlichen Disziplinen und Forschungen, erläutert Charakterzüge der Stadt aus den „großen Erzählungen“ zentraler Autorinnen/Autoren und vermittelt ein Bild von der Stadt vor dem Hintergrund unterschiedlicher Sprachen. Alle drei Zugänge werden am Ende jeweils in einem Abschnitt noch einmal zusammengefasst und damit noch fokussierter festgehalten. Die Quintessenz aus den disziplinären Darstellungen der Stadt - z. B. der Urbanistik (,Das Große Ganze“), der Soziologie und Stadtsoziologie (,Stadt als Gesellschaft“), der Raumplanung, Stadtplanung, Architektur und Stadtmorphologie (,Stadt als Gestaltungsraum“), aber auch der Rechtswissenschaften (,Stadt als Regelwerk“) - ist die Stadt als Suchbild. Die Zusammenfassung des zweiten Zugangs, der großen Erzählungen (von Aristoteles über die Chicago School bis hin zu Sassen und Sennett), kommt dagegen mit dem recht nüchternen Titel „Erkenntnisse der Expertinnen und Experten“ aus. Der dritte Zugang über verschiedene Sprachen und die ihnen innewohnende Bedeutung von Stadt schließt wiederum mit einer Überschrift, die einerseits auf die unterschiedlichen Ursprünge von Stadt verweist und 
andererseits die Grundlage ihres Wesens bezeichnet: „Die Gene der Stadt".

In dem zweiten umfangreicheren Kapitel zur Praxis werden Stadtbegriffe, Stadtkonzepte und Stadttypen vorgestellt. Prell analysiert, welche der von ihm ausgewählten acht Funktionen den jeweiligen Konstruktionen, wie z. B. der Global City, zugeschrieben werden. Wird mit ihnen etwa eine bestimmte Programmatik oder ein Leitbild verfolgt? In einem zweiten Schritt fragt er nach der Ausprägung der zuvor benannten Merkmale wie der Verdichtung, um dann in einem dritten Schritt eine Antwort auf die Leitfrage des Buches (z. B. „Auf welche Frage antwortet die Global City?") zu formulieren. Über ein Punktesystem gewichtet Prell die Funktionen und Ausprägungen der jeweiligen Stadttypen und versucht so, einen Vergleichsmaßstab herzustellen. Hierin ist auch eine Übersicht zu der Frage „Auf welche Frage bietet ein Stadttyp eine Antwort?" (S. 119) enthalten, in der beispielsweise die Antwort der Global City die „hochspezialisierte Kommando-/Steuerungszentrale“ ist.

Schließlich widmet sich Prell einigen zentralen Themen (,Stadtthemen“) wie der Zuwanderung und Abwanderung, dem Wohnen und Leben, der Bewegung und dem Stillstand (Stichwort: Mobilität) oder der Digitalisierung. Er verknüpft auch dies immer wieder mit den zuvor genannten Stadttypen und -konzepten, stellt Querverbindungen her, die insgesamt verdeutlichen, dass es um eine Darstellung der verschiedenen Zugänge zu und Perspektiven auf die Stadt geht.

Eine eindeutige Aufforderung, sich mit Stadt zu beschäftigen, ist schließlich die Überschrift des letzten Kapitels des Buches: „Unsere Zukunft entscheidet sich in und mit der Stadt“. Mit diesem Kapitel schließt sich der Kreis. Denn gleich zu Beginn des Buches stellt Prell fest, dass derzeit mehr als die Hälfte der Menschen in der Stadt leben, diese Form des Zusammenlebens (oder der „Antwort“) sehr gefragt ist. Entsprechend ist auch unsere Zukunft eng mit ihr verbunden. Eine Form, gekennzeichnet durch Vielfalt/ Heterogenität und Infrastruktur, ist vor allem jedoch - und dies bezeichnet Prell als Alleinstellungsmerkmal der Stadt Verdichtung. In Anlehnung an den Kreativitätsbegriff von Joas (1992) konstatiert Prell, dass kreatives Handeln unter der Bedingung der Verdichtung zu Ergebnissen führt, die nur in der Stadt möglich seien (vgl. S. 80 und S. 135). Und weiter: dass ,die Stadt immer noch das beste Werkzeug zur Steigerung der menschlichen Möglichkeiten im Kollektiv ist“ (S. 137). Rückblickend auf die Leitfragen des Buches bedeutet dies, dass die Stadt die Antwort auf die Herausforderungen und Fragen des 21. Jahrhunderts zu sein scheint, die nur im Kollektiv und kreativ zu lösen sind. Das entspricht einer gänzlich anderen Sichtweise auf die Stadt, als sie in vielen Fachdiskursen und Perspektiven auf die Stadt vertreten wird: Hier sind die Probleme und Herausforderungen der Gesellschaft der Ausgangspunkt, die sich in der Stadt wie in einem Brennglas verdichten.

Insofern beinhaltet das Buch ein eindeutiges Plädoyer für die Stadt. Es bietet über die umfangreich ausgewählte, pointiert und mit vielen Bezügen vorgestellte Literatur einen guten sozialwissenschaftlichen Überblick über das, was in der Vergangenheit und aktuell zur Stadt geschrieben wurde und wird. Tabellen und der stetige Bezug zu den durch die Disziplinen oder Autorinnen/Autoren ins Zentrum gesetzten Eigenschaften der Stadt (wie die Dichte) schaffen eine sehr gute Übersicht über die Ansätze und bieten gleichzeitig eine klare Struktur - wenn auch die im vierten Kapitel „Praxis“ vorgenommene Quantifizierung und Gewichtung über ein Punktesystem weniger nachzuvollziehen ist. Was hier und da irritiert und gleichzeitig eine gewisse Faszination erzeugt, ist die ungewöhnliche Sprache, in der die Überlegungen präsentiert werden (z. B. Form und Werkzeug für die Stadt). Dies braucht eine gewisse Gelassenheit gegenüber Begriffen - die manchen Studierenden mit dem Bedürfnis nach klaren Definitionen fehlen könnte. Gleichzeitig regt der bildreiche Stil sehr an, sich selbst Gedanken über konkrete Städte zu machen - so etwa wenn es um die Vergleichbarkeit einer antiken Stadt mit der Smart City geht. Die Nicht-Vergleichbarkeit, so stellt Prell fest, ist einerseits banal. „Andererseits stimmt diese Aussage schlicht nicht, denn wir hätten nicht das geringste Problem, etwa eine Stadt wie das 79 n. Chr. beim Ausbruch des Vesuv verschüttete und erst in der Neuzeit wieder ausgegrabene Pompeji binnen Stunden in Betrieb zu nehmen und mit urbanem Leben zu füllen, Infrastruktur inklusive, vom Streetfood-Stand bis zum WLAN“ (S. 13). Die bildhafte Sprache, die Vergleiche und hergestellten Bezüge zwischen der Vergangenheit und der Gegenwart ebenso wie der Bezug auf konkrete Städte machen das Buch sehr lesenswert (und sind keinesfalls belehrend).

Prell legt mit seinem Buch „Die Stadt“ eine interdisziplinäre Arbeit vor, die mit dem Untertitel „Einführung in die Sozialwissenschaften" auch Studierende aus unterschiedlichen Disziplinen ansprechen soll. Der Anspruch ist damit hoch, denn es gilt, an Fachdiskurse der (Stadt-)Soziologie ebenso wie der (Stadt-)Geographie und weiterer anzuknüpfen. Obwohl Prell diesem Anspruch nur zum Teil genügen kann, da seine Bezüge insgesamt doch eher soziologischer Natur sind, sind seine Überlegungen (als urban studies) für viele die Stadt behandelnden Disziplinen anschlussfähig. Dies liegt vor allem an dem beschriebenen Konzept des Buches. Es ist eben kein Handbuch, wie das von Harald Mieg und Christoph Heyl im Jahr 2013 herausgegebene „Stadt: ein interdisziplinäres Handbuch“ (Mieg/ Heyl 2013) oder - und hier bietet sich ein Vergleich an wie das zeitgleich von Ingrid Breckner, Albrecht Göschel 
und Ulf Matthiesen mit der Unterstützung vieler Autorinnen und Autoren herausgegebene Sammelwerk ,Stadtsoziologie und Stadtentwicklung. Ein Handbuch für Wissenschaft und Praxis“ (Breckner/Göschel/Matthiesen 2020). Die Empfehlung in Bezug auf dieses Sammelwerk wäre, zunächst „Die Stadt" von Uwe Prell zu lesen, um in dem Handbuch dann Aspekte zu vertiefen und Weiteres hinzuzunehmen.

Vollständige bibliographische Angaben des rezensierten Werkes:

Prell, Uwe (2020): Die Stadt. Eine Einführung für die Sozialwissenschaften. Opladen/Toronto: Verlag Barbara Budrich. 148 Seiten

\section{Literatur}

Breckner, I.; Göschel, A.; Matthiesen, U. (Hrsg.) (2020): Stadtsoziologie und Stadtentwicklung. Handbuch für Wissenschaft und Praxis. Baden-Baden.

Joas, H. (1992): Die Kreativität des Handelns. Frankfurt am Main.

Mieg, H.A.; Heyl, C. (Hrsg.) (2013): Stadt: ein interdisziplinäres Handbuch. Stuttgart. 